\title{
HUERTOS MELÍFEROS CON ESPECIES FORESTALES NATIVAS UNA ALTERNATIVA PARA APOYAR A LA AGRICULTURA FAMILIAR CAMPESINA Y MEJORAR EL NEGOCIO APÍCOLA ${ }^{5}$
}

Molina, M. P.; Soto, H.; Gutiérrez, B.; González, J.; Koch, L.; Ipinza, R.; Rojas, P. y Chung, P. ${ }^{6}$

\section{RESUMEN}

El Instituto Forestal, a través del equipo de trabajo de Conservación y Mejoramiento Genético, se encuentra ejecutando un programa, apoyado por el Gobierno Regional del Bio Bio, que tiene como objetivo aumentar la cantidad de flores melíferas disponibles a través de la restauración y suplementación de la diversidad floral, con énfasis en especies forestales nativas y entregando, además, herramientas tecnológicas a los apicultores para enfrentar fenómenos climáticos o de disponibilidad que afecten la producción de la miel y sus derivados.

El modelo de trabajo propuesto y el diseño de las unidades melíferas son aplicables a otras regiones geográficas del país y conlleva adicionalmente la restauración y conectividad biológica de la flora nativa chilena.

Se presentan las bases del programa y los avances a 1,5 años de su puesta en marcha $y$, adicionalmente se agregan antecedentes para su aplicación más al sur en la Patagonia chilena.

Palabras clave: Huertos melíferos, Especies nativas, Diversidad floral

\section{SUMMARY}

The Chilean Forestry Institute's Conservation and Genetic Improvement Group is currently executing a program related to apiculture, which is financed by the Bio Bio Regional Government. The program's objective is to increase the number of available flowers through the restoration and supplementation of floral diversity with emphasis in native forest species. The program also tries to deliver technological tools to beekeepers to make them able to deal with the climatic variables that affect honey and derivative production.

Both, the proposed working model and the design of the honey units are applicable to other geographic regions of the country. In addition, this scheme contributes to the restoration and biological connectivity of the native Chilean flora.

The bases and progress of the program after 1.5 years since its implementation are presented in this paper. Some background information for its application in the Chilean Patagonia is presented as well.

Key words: Honey orchards, Native species, Flower diversity.

\footnotetext{
${ }^{5}$ Trabajo presentado en forma resumida al Congreso Internacional Agroforestal y Forestal Patagónico realizado en Puerto Natales, Chile. Diciembre 2016.

${ }^{6}$ Investigadores Instituto Forestal, Sedes Metropolitana, Santiago; Bio Bio, Concepción y Los Ríos, Valdivia. mmolina@infor.cl; hsoto@infor.cl; bgutierr@infor.cl; jgonzalez@infor.cl; Ikoch@infor.cl; roberto.ipinza@infor.cl; parojas@infor.cl; pchung@infor.cl.
} 


\section{INTRODUCCIÓN}

El Instituto Forestal ha estado sistemáticamente unido al desarrollo de la pyme chilena, a través de trabajo colaborativo con asociaciones gremiales de pequeños y medianos propietarios, como ProNitens, productores de Eucalyptus nitens; APROBOSQUE, propietarios de Bosque Nativo, y más recientemente con el trabajo coordinado con PYMEMAD, que es la agrupación de pymes madereras.

En el ámbito de la conservación de recursos genéticos forestales chilenos, INFOR promueve el uso del modelo circa situm (conservar haciendo), popularizado por David Boshier, profesor de la Universidad de Oxford. Este modelo se ha aplicado en el ámbito de la creación de huertos melíferos con especies nativas. Estos huertos, verdaderos bancos de conservación de recursos genéticos forestales, se establecen y se usan con los apicultores asociados a BIOMIEL AG, para disponer de una mejor oferta de néctar y polen en apoyo a la producción de miel de la pyme apícola regional. De esta forma, los huertos melíferos son valorados y se convierten en verdaderas unidades de conservación ex situ.

Según datos del VII Censo Agropecuario del año 2007, en Chile existen alrededor de 10 mil explotaciones (predios) que administran más de 454 mil colmenas (ODEPA, 2016) y producen miel y otros productos, como cera, propóleo, polen, jalea real y otros, conformando una variada gama de productos apícolas como cadena productiva. La producción de miel, originada en parte en especies melíferas del bosque nativo chileno, es exportada en cerca de un $90 \%$ a los mercados de la Unión Europea y Estados Unidos, constituyéndose en el principal producto pecuario primario exportado por Chile (FIA, 2016).

La actividad melífera en Chile enfrenta una considerable pérdida de competitividad, consecuencia de una importante reducción de su producción, debida a causas conjugadas que incluyen desde años de sequía hasta procesos históricos de deforestación y cambios de uso de suelos forestales a agropecuarios. A juicio de los propios apicultores, especialmente los de la región del Bio Bio, la escasez de flora melífera y la irregularidad en los flujos de néctar son las principales causas de esta disminución productiva; mientras que la falta de diferenciación de sus mieles es uno de los principales obstáculos para su comercialización. La pérdida de competitividad de la apicultura de la región se ve reflejada en una importante disminución de la participación en la producción nacional de mieles, bajando aproximadamente desde un $46 \%$ en la producción nacional hasta un $21 \%$ durante el año 2013 , año en el que se formula una propuesta al Gobierno Regional del Bio Bio para buscar soluciones técnicas al problema.

Se reconoce la existencia de especies forestales nativas con destacadas características melíferas, capaces de generar mieles muy valoradas por el mercado. Sin embargo, la participación de estas especies en la oferta de flores para apicultura ha ido disminuyendo gradualmente debido al fuerte proceso de deterioro y degradación que ha experimentado el bosque nativo. Adicionalmente, estas especies no suelen cultivarse y no existen conocimientos suficientes para propagarlas, establecerlas y manejarlas con la finalidad de constituir una fuente sustentable de alimentación para la producción apícola. Urge generar alternativas productivas que contribuyan al uso sustentable del bosque, disminuyéndose con ello la degradación y deterioro de un recurso que en muchos casos se ha visto afectado por prácticas de extracción de leña, la habilitación agropecuaria e incluso la urbanización.

Se suma a lo anterior que la mayor parte de los apicultores desconoce las técnicas necesarias para la propagación y plantación de especies forestales melíferas y el manejo de la flora existente, por lo cual, en muchos casos, no tienen las condiciones mínimas para enfrentar cambios en la producción de flores y néctar para el negocio de las mieles y sus derivados.

En este contexto, en el proyecto desarrollado en el Bio Bio se propone como unidad tecnológica el establecimiento de huertos melíferos definidos como "una plantación mixta de árboles, arbustos y hierbas con especies de gran aptitud melífera, orientada a la producción de néctar y polen con una amplia ventana de floración". Las plantas pueden ser originadas por 
semillas o por propágulos vegetativos (estacas o injertos), que florezcan la mayor parte del año, y deben ser manejadas intensivamente para la producción de flores (fertilización, podas de formación de copas, riegos, control de malezas, entre otras prácticas).

El presente documento describe los objetivos de este proyecto y las acciones y resultados alcanzados a la fecha en la región del Bio Bio y, complementariamente, se incluye una aproximación a lo que podría ser la aplicación de una iniciativa de similares características en la Patagonia chilena.

\section{OBJETIVOS}

\section{Objetivo General}

Aumentar la cantidad de flores melíferas a través del manejo y suplementación de la diversidad floral, con énfasis en especies forestales nativas.

\section{Objetivos Específicos}

Mejorar la composición florística de sectores apícolas de la Región del Bio Bio.

Capacitación en producción de flores para la apicultura.

Caracterización preliminar de la miel regional

\section{MATERIAL Y MÉTODO}

\section{Mejorar la Composición Florística de Sectores Apícolas de la Región del Bio Bio}

Parte de la solución al problema planteado para la región del Bio Bio; pérdida de competitividad y producción decreciente o irregular, fue abordada a través del mejoramiento de la producción predial de flores, involucrando por una parte el manejo de bosquetes nativos existentes y la generación de nuevos bosquetes melíferos con especies nativas melíferas establecidas especialmente para estos fines, y por otra suplementando la producción de flores melíferas, hasta la entrada en producción de las unidades anteriores, con la plantación de especies arbóreas de rápido crecimiento. Esto además de otras opciones propuestas por los apicultores que son de uso común en las labores agrícolas, tales como el uso de las praderas, reconocidas como productoras de flores de uso melífero.

El proyecto contempla la aplicación de estas técnicas basándose en un ordenamiento predial que no excluya otras actividades, también relevantes para la calidad de vida de los propietarios. En este aspecto la acción participativa es fundamental. Se detectan preferencias por distintas especies forestales melíferas y ese es el principal criterio utilizado para la selección de las mismas. Dada la variabilidad climática y edáfica en la región, es importante que INFOR, como organismo de investigación del Estado, oriente al apicultor en las ventajas y desventajas de la elección y manejo de algunas especies.

En tal contexto, el proceso se inició con la colaboración de la asociación de apicultores más importante de la región, Biomiel AG, la que aportó su nómina de asociados a quienes en distintas oportunidades se les presentó el proyecto. Se detallaron los requisitos para participar en el mismo según la normativa del Gobierno Regional del Bio Bio. En este caso, los requisitos fundamentales eran ser apicultor (sobre el $50 \%$ de sus ingresos debía provenir de la apicultura) y ser propietario del predio donde se establecerían las unidades melíferas.

En reuniones entre los apicultores involucrados y el equipo del proyecto se definieron las principales especies melíferas de acuerdo a lo observado y preferido por los apicultores. De las 
especies seleccionadas se compiló información relevante de época de floración, de producción de semillas, de protocolos de producción de plantas, de requerimientos ecológicos y de toda aquella información que contribuyera a conseguir plantas de calidad que asegurarán su supervivencia en terreno.

En otra etapa del proyecto se ha propuesto también la realización de calendarios de floración de las especies de utilidad melífera presentes en cada uno de los predios de los apicultores de modo de manejar la fecha de cosecha de las colmenas para la obtención de mieles diferenciadas.

Con la información reunida y llegada la época se procedió a cosechar semilla de las especies propuestas y se dio inicio a la producción de plantas en el vivero institucional, en el cual paralelamente se acondicionaron plantas de algunas de estas especies elegidas que estaban como remanentes de producción de años anteriores. En general estas plantas se encontraban en contenedores de baja capacidad volumétrica de sustrato (135 a $180 \mathrm{cc}$ ) por lo cual para poder utilizarlas en los primeros huertos melíferos se procedió a traspasarlas a bolsas de $2,5 \mathrm{~L}$ con sustrato tradicional (corteza de pino compostada) enriquecido con fertilizantes de lenta entrega con NPK.

Considerando la duración del proyecto (3 años) y el desconocimiento empírico del protocolo de producción de plantas de la mayoría de las especies recolectadas, se extrajo semilla de al menos 5 árboles por especie, hasta el 60\% aproximadamente de la semilla producida por cada árbol, con el fin de obtener el mayor número posible de plantas para abastecer todo el periodo de ejecución del programa, incluyendo su eventual ampliación de tres años.

Con posterioridad al inicio de las actividades descritas se inició la validación de sitios donde se establecerían las unidades melíferas. Los comités comunales o asociaciones integrantes de Biomiel AG consultaron a sus miembros respecto de su interés por contar con una unidad melífera y por el compromiso que ellos podían asumir para su mantención.

Se preparó una lista de propietarios preseleccionados y se visitó cada uno de los terrenos disponibles de modo de evaluar la factibilidad técnica (sin anegamiento, suelo con estructura, pendientes moderadas, entre otras características) y determinar qué necesidades de preparación de suelo se requerían, además, de qué especies forestales eran las más apropiadas de acuerdo a los requerimientos ecológicos de estas y el interés del propietario. En muchos casos existía preferencia de los propietarios por especies más reconocidas, pero que no necesariamente eran las adecuadas para su terreno, de modo que fue necesario disponer de los antecedentes ecológicos de las especies de modo de señalar las desventajas y ventajas de utilizar cada una de ellas en cada caso.

Una vez visitados los terrenos candidatos, fueron descartados aquellos que no cumplían con los requisitos administrativos y técnicos.

Cabe señalar que la mayor parte de los apicultores son también agricultores, que utilizan sus suelos con diversos cultivos, tales como raps, trigo, papas entre otros, y en algunos casos también realizan ganadería de ovinos. En general son propiedades pequeñas, todas de menos de 50 hectáreas y hasta 2 hectáreas, y con suelos muy compactados ("pie de arado").

En el año 2015 la preparación de los suelos se inició bastante tarde en el mes de junio, finalizándose la plantación en agosto. El año 2016 se realizó la preparación de suelo entre los meses de abril y mayo, y la plantación se efectuó entre junio y la primera quincena de septiembre. La región tiene un déficit de precipitaciones cercano al 24\% en los últimos 9 años por lo cual se decidió realizar una preparación intensiva del suelo de acuerdo a los recursos existentes y el desarrollo de un sistema de plantación que asegurara la supervivencia de las plantas.

Dentro de los sistemas de preparación de suelo, el subsolado de las hileras de plantación es el más efectivo para recuperar suelos compactados por el uso agrícola o bien por el ganadero, 
sin embargo, su aplicación depende de la pendiente del terreno, de la disponibilidad de maquinaria y de la superficie a intervenir. En relación a esto último, en muchos casos la reducida superficie a trabajar (0,5 a 1 ha) no hacía factible el subsolado, dado que el trabajo no resultaba atractivo para terceros que brindan el servicio. Alternativamente, se utilizó también un barreno para ahoyar con motor de 2 tiempos, equipo de uso manual que permitió en la mayoría de los casos efectuar las casillas donde se ubicarían las plantas. En ambos casos, previo a la plantación se confeccionaron casillas de 50 × 50 × $50 \mathrm{~cm}$ y se realizó control manual de malezas en la hilera de plantación. En el terreno restante se mantuvieron las herbáceas y arbustivas que tenían utilidad melífera.

La plantación propiamente tal consideró a las especies idóneas para cada sector, combinado al menos 13 a 15 de ellas en cada unidad. El espaciamiento utilizado fue de $4 \times 4$ o $5 \times$ $5 \mathrm{~m}$, dependiendo de la disponibilidad de terreno. Cada una de las plantas fue establecida con $3 \mathrm{~g}$ de gel hidratante, $30 \mathrm{~g}$ de fertilizante de lenta entrega, en este caso de 9 meses de duración, con NPK 16:8:12. Las plantas fueron protegidas individualmente con malla Raschel ( $1 \mathrm{~m}$ de altura $\times 40$ $\mathrm{cm}$ de diámetro y $80 \%$ de capacidad de sombreamiento), con el fin de evitar el exceso de insolación y generar un microclima en torno a la planta más adecuado para su desarrollo; adicionalmente esta protección es efectiva para controlar malezas alrededor de la planta y para protegerla de lagomorfos o del ramoneo de ganado menor. Este sistema de protección tiene una duración aproximada de 5 años, pero se espera mantenerlo solo los 2 primeros años de la plantación hasta que la planta haya superado en un $100 \%$ la altura de la malla. Una vez finalizado este proceso las protecciones pueden ser reutilizadas.

Durante el periodo estival posterior a la plantación del año 2015, se regó los huertos con $5 \mathrm{~L}$ de agua por planta y se realizó control de maleza en aquellas unidades que lo requerían. En los huertos establecidos en zonas de secano se realizó un riego adicional. Esta labor debería llevarse a cabo también los 2 primeros años post-plantación. En general los terrenos no cuentan con riego por lo que se utiliza un estaque de $1.000 \mathrm{~L}$ adosado a una camioneta y una bomba que permite por una parte llenar este estanque desde alguna fuente (pozo, estero, río) y también distribuir el agua en la plantación misma si el terreno lo permite.

\section{Capacitación en Producción de Flores para la Apicultura}

Todas las técnica ya implementadas y por implementar durante el desarrollo del proyecto serán transferidas en forma teórica y práctica a los apicultores involucrados, esto con el fin que ellos puedan replicar estas prácticas en otros predios y difundirlas a otros apicultores. El fin es generar monitores que extiendan conocimiento a sus pares. Se busca así que efectivamente los resultados tengan el impacto suficiente para que otros apicultores adopten las soluciones propuestas, se generen unidades de servicios tales como viveros forestales destinados a la producción de plantas melíferas, surjan especialistas en el manejo forestal con fines apícolas, y en última instancia que el grupo objetivo tenga las herramientas necesarias para revertir la carencia de flores melíferas que afectan la sustentabilidad de su negocio.

Fueron diseñados dos cursos teórico-prácticos de injertación y producción de plantas con utilidad melífera, ambos desarrollados en las instalaciones de INFOR. Igualmente, durante el periodo de plantaciones, se programaron capacitaciones directas en terreno, de modo que los apicultores realizaran esta labor en forma práctica.

\section{Caracterización Preliminar de la Miel Regional}

Se encargaron análisis fisicoquímicos de 61 muestras de mieles de distintos apiarios de la región en las cuales se evaluaron cinco tipos de parámetros relacionados con:

-Madurez o sazón de la miel, tales como humedad, índice de refracción, sólidos totales, peso específico, sacarosa aparente.

-Manejo posterior a la cosecha, evaluándose hidroximetil furfural (HMF). 
-Caracterización de la miel; pH, color, conductividad, ceniza, azúcares reductoras (glucosa, fructosa, manosa y otros azúcares mono, di y trisacáridos).

-Frescura de la miel; índice de diastasa.

-Grado de antioxidantes de la miel; índice del contenido de compuestos fenólicos e índice del contenido de compuestos antioxidantes filtradores de la luz UV

Estos resultados serán correlacionados posteriormente con análisis botánicos melisopalinológicos, que también fueron encargados para estas mieles, los cuales determinan a través del polen su origen florístico.

\section{RESULTADOS Y DISCUSIÓN}

\section{Mejorar la Composición Florística de Sectores Apícolas de la Región del Bio Bio}

Biomiel AG puso a disposición un listado de sus socios según Comuna (Cuadro № 1) y en diversas oportunidades los convocó para que conocieran los antecedentes del proyecto, analizaran su inserción dentro de las acciones planificadas y participaran en la selección de especies melíferas.

Cuadro $\mathrm{N}^{\circ} 1$

DISTRIBUCIÓN POR COMUNA DE APICULTORES DE LA ASOCIACIÓN BIOMIEL A.G. Y USUARIOS SAT

\begin{tabular}{|c|c|c|c|c|c|c|c|}
\hline \multirow{2}{*}{ Comuna } & Hombres & Mujeres & Total & Comuna & Hombres & Mujeres & Total \\
\hline & \multicolumn{7}{|c|}{$\left(\mathrm{N}^{\circ}\right)$} \\
\hline \multicolumn{4}{|c|}{ 1.- Organizaciones Socias Biomiel } & \multicolumn{4}{|c|}{ 2.- Usuarios Servicio Asesoría Técnica (SAT) } \\
\hline Concepción & 52 & 13 & 65 & Bulnes & 5 & 1 & 6 \\
\hline Chillán & 11 & 6 & 17 & Quillón & 6 & 3 & 9 \\
\hline Quillón & 10 & 1 & 11 & Yumbel & 11 & 9 & 20 \\
\hline Quirihue & 5 & 6 & 11 & Cabrero & 2 & 4 & 6 \\
\hline Sta. Bárbara & 4 & 3 & 7 & Chillan & 6 & 3 & 9 \\
\hline Cañete & 17 & 4 & 21 & Chillan Viejo & 4 & & 4 \\
\hline San Nicolás & 10 & 4 & 14 & Coihueco & 8 & 3 & 11 \\
\hline Ranquil & 12 & 9 & 21 & Pinto & 1 & 2 & 3 \\
\hline Florida & 6 & 14 & 20 & Portezuelo & 1 & - & 1 \\
\hline Chillan Viejo & 5 & 8 & 13 & San Nicolás & 5 & 3 & 8 \\
\hline Los Álamos & 7 & 5 & 12 & Ñiquén & 5 & 1 & 6 \\
\hline Hualqui & 24 & 12 & 36 & San Carlos & 2 & 2 & 4 \\
\hline El Carmen & 28 & 4 & 32 & San Fabián & 5 & 3 & 8 \\
\hline Total 1 & 191 & 89 & 280 & Cobquecura & 1 & & 1 \\
\hline & & & & Ninhue & 6 & 2 & 8 \\
\hline & & & & Quirihue & 5 & 2 & 7 \\
\hline & & & & Total 2 & 73 & 38 & 111 \\
\hline & & & & TOTAL 1+2 & 264 & 127 & 391 \\
\hline
\end{tabular}

En conjunto con los apicultores de la región se definieron las especies melíferas que ellos estimaban eran las con mejores resultados para la producción de miel. Para tales especies se recogió información respecto de su época de floración, producción de semillas, distribución geográfica y la función de su flor en el ciclo biológico de las abejas (Cuadro № 2). 
Cuadro $\mathbf{N}^{\circ} 2$

ESPECIES MELÍFERAS PRESENTES EN LA REGIÓN DEL BIO BIO, ÉPOCA DE FLORACIÓN, DE PRODUCCIÓN DE SEMILLAS Y SU UTILIDAD PARA EL CICLO BIOLÓGICO DE LAS ABEJAS

\begin{tabular}{|c|c|c|c|c|c|c|c|c|c|c|c|c|c|c|c|}
\hline $\begin{array}{l}\text { Nombre } \\
\text { Común }\end{array}$ & $\begin{array}{l}\text { Nombre } \\
\text { Cientifico }\end{array}$ & \multicolumn{13}{|c|}{ CALENDARIZACIÓN Y DISTRIBUCIÓN GEOGRÁFICA } & $\begin{array}{l}\text { Tipo de Planta-Ciclo } \\
\text { Reproductivo Abeja }\end{array}$ \\
\hline \multirow{3}{*}{ Quillay } & \multirow{3}{*}{$\begin{array}{c}\text { Quillaja } \\
\text { saponaria }\end{array}$} & $\mathbf{F}$ & E & $\mathrm{F}$ & M & A & $\mathrm{M}$ & a & a & A & s & $\circ$ & $\mathrm{N}$ & D & \multirow{3}{*}{$\begin{array}{l}\text { Plantas de producción de } \\
\text { excedentes }\end{array}$} \\
\hline & & $\mathrm{c}$ & $E$ & $\mathrm{~F}$ & M & A & $\mathrm{M}$ & J & 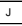 & $\mathrm{A}$ & s & $\circ$ & $\mathrm{N}$ & $\mathrm{D}$ & \\
\hline & & D & \multicolumn{12}{|c|}{ Coquimbo - La Araucania } & \\
\hline & & $\mathbf{F}$ & $\mathrm{E}$ & $\mathrm{F}$ & M & A & $\mathrm{M}$ & J & 7 & A & s & 0 & $\mathrm{~N}$ & D & \multirow{3}{*}{$\begin{array}{l}\text { Plantas de mantención y } \\
\text { desarrollo }\end{array}$} \\
\hline Maqui & $\begin{array}{l}\text { Anstotella } \\
\text { chilensis }\end{array}$ & c & E & $\mathrm{F}$ & M & A & $\mathrm{M}$ & $\mathrm{J}$ & a & $\mathrm{A}$ & s & $\circ$ & $\mathrm{N}$ & $\mathrm{D}$ & \\
\hline & & $\mathrm{D}$ & & & & & & quim & Ays & & & & & & \\
\hline & & $\mathbf{F}$ & $\mathrm{E}$ & $\mathrm{F}$ & M & A & $\mathrm{M}$ & a & $\mathrm{a}$ & A & $\mathrm{s}$ & $\circ$ & $\mathrm{N}$ & D & \\
\hline Uimo & $\begin{array}{l}\text { Eucryyhia } \\
\text { cordifolia }\end{array}$ & $\mathrm{c}$ & $E$ & $\mathrm{~F}$ & M & A & $\mathrm{M}$ & a & 2 & A & $\mathrm{s}$ & 0 & $\mathrm{~N}$ & D & $\begin{array}{l}\text { Plantas depProducción de } \\
\text { excedentes }\end{array}$ \\
\hline & & D & & & & & & Bio & s Lag & & & & & & \\
\hline & & $\mathbf{F}$ & $\mathrm{E}$ & $\mathrm{F}$ & M & A & $\mathrm{M}$ & J & j & A & $\mathrm{s}$ & 0 & $\mathrm{~N}$ & D & Plantas de estimulación, plantas \\
\hline Canelo & $\begin{array}{c}\text { Drimys } \\
\text { winteri }\end{array}$ & c & $E$ & $\mathrm{~F}$ & M & A & M & J & 2 & $\mathrm{~A}$ & s & $\circ$ & $\mathrm{N}$ & $\mathrm{D}$ & $\begin{array}{l}\text { de mantención y desarrollo } \\
\text { plantas de producción de }\end{array}$ \\
\hline & & D & & & & & & imb & os $L$ & & & & & & \\
\hline & & $\mathbf{F}$ & $E$ & $\mathrm{~F}$ & M & A & $\mathrm{M}$ & $\mathrm{da}$ & a & A & $s$ & 0 & $\mathrm{~N}$ & $D$ & \\
\hline Maitén & $\begin{array}{c}\text { Maytenus } \\
\text { boaria }\end{array}$ & c & $E$ & $\mathrm{~F}$ & M & A & $\mathrm{M}$ & 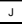 & J & $\mathrm{A}$ & $\mathrm{s}$ & $\circ$ & $\mathrm{N}$ & $\mathrm{D}$ & $\begin{array}{l}\text { Plantas de estimulación, plantas } \\
\text { de mantención y desarrollo }\end{array}$ \\
\hline & & D & & & & & & $\mathrm{ca}-1$ & allan & & & & & & \\
\hline & & $\mathbf{F}$ & $\mathrm{E}$ & $\mathrm{F}$ & M & A & $\mathrm{M}$ & J & a & A & $\mathrm{s}$ & 0 & $\mathrm{~N}$ & D & \\
\hline Radal & $\begin{array}{l}\text { Lomatia } \\
\text { hirsuta }\end{array}$ & $\mathrm{c}$ & $E$ & $\mathrm{~F}$ & M & A & $\mathrm{M}$ & J & 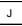 & $\mathrm{A}$ & $\mathrm{s}$ & $\circ$ & $\mathrm{N}$ & $\mathrm{D}$ & $\begin{array}{l}\text { Plantas de estimulación, plantas } \\
\text { de mantención y desarrollo }\end{array}$ \\
\hline & & D & & & & & & imb & os $L$ & & & & & & \\
\hline & & $\mathbf{F}$ & $\mathrm{E}$ & $\mathrm{F}$ & M & A & $\mathrm{M}$ & a & $\mathrm{a}$ & A & $\mathrm{s}$ & $\circ$ & $\mathrm{N}$ & D & \\
\hline Boldo & $\begin{array}{l}\text { Peumus } \\
\text { boldus }\end{array}$ & $\mathrm{c}$ & $E$ & $\mathrm{~F}$ & M & A & $\mathrm{M}$ & J & $\mathrm{s}$ & $\mathrm{A}$ & $\mathrm{s}$ & $\circ$ & $\mathrm{N}$ & D & Plantas de estimulación \\
\hline & & D & & & & & & imb & os $L$ & & & & & & \\
\hline & & $\mathbf{F}$ & $E$ & $\mathrm{~F}$ & M & A & $\mathrm{M}$ & J & 2 & A & $s$ & 0 & $\mathrm{~N}$ & D & Plontra d astimuccín \\
\hline Avellana & $\begin{array}{l}\text { Corylus } \\
\text { columa }\end{array}$ & $\mathrm{c}$ & $E$ & $\mathrm{~F}$ & M & A & $\mathrm{M}$ & 2 & a & A & $\mathrm{s}$ & $\circ$ & $\mathrm{N}$ & D & Iantas de estimulacion \\
\hline & & D & & & & & & $10-4$ L & rauce. & & & & & & \\
\hline & & $\mathbf{F}$ & $\mathrm{E}$ & $\mathrm{F}$ & M & A & $\mathrm{M}$ & $\mathrm{a}$ & a & A & $\mathrm{s}$ & $\circ$ & $\mathrm{N}$ & D & \\
\hline Madroño & $\begin{array}{c}\text { Escallonia } \\
\text { oulverulenta }\end{array}$ & c & $E$ & $\mathrm{~F}$ & M & A & $\mathrm{M}$ & J & 2 & A & $s$ & 0 & $\mathrm{~N}$ & D & $\begin{array}{l}\text { Plantas de producción de } \\
\text { excedentes }\end{array}$ \\
\hline & & D & & & & & & imb & os $L$ & & & & & & \\
\hline & & $\mathbf{F}$ & $\mathrm{E}$ & $\mathrm{F}$ & M & A & $\mathrm{M}$ & $\mathrm{J}$ & J & A & $\mathrm{s}$ & $\circ$ & $\mathrm{N}$ & D & \\
\hline Guindo Santo & $\begin{array}{l}\text { Eucryphia } \\
\text { glutinosa }\end{array}$ & c & $\mathrm{E}$ & $\mathrm{F}$ & M & A & $\mathrm{M}$ & a & $\mathrm{a}$ & A & $\mathrm{s}$ & $\circ$ & $\mathrm{N}$ & D & $\begin{array}{l}\text { Plantas de producción de } \\
\text { excedentes }\end{array}$ \\
\hline & & D & & & & & & $10-5$ & rauce. & & & & & & \\
\hline & & $\mathbf{F}$ & $E$ & $\mathrm{~F}$ & M & A & $\mathrm{M}$ & $\mathrm{d}$ & $\mathrm{a}$ & A & $s$ & 0 & $\mathrm{~N}$ & D & \\
\hline Peumo & $\begin{array}{c}\text { Cryptocarya } \\
\text { alba }\end{array}$ & c & $\mathrm{E}$ & $\mathrm{F}$ & M & A & $\mathrm{M}$ & J & a & A & $\mathrm{s}$ & $\circ$ & $\mathrm{N}$ & D & $\begin{array}{l}\text { Plantas de estimulación, plantas } \\
\text { de mantención y desarrollo }\end{array}$ \\
\hline & & D & & & & & & uimb & Los & & & & & & \\
\hline & & $\mathrm{F}$ & $\mathrm{E}$ & $\mathrm{F}$ & M & A & $\mathrm{M}$ & ᄀ & ᄀ & $\mathrm{A}$ & $s$ & $\circ$ & $\mathrm{N}$ & $\mathrm{D}$ & \\
\hline Robinia o Acacio & $\begin{array}{l}\text { Robinia } \\
\text { pseudoacacia }\end{array}$ & $\mathrm{c}$ & $E$ & $\mathrm{~F}$ & M & A & $\mathrm{M}$ & $\mathrm{d}$ & $\mathrm{d}$ & A & $\mathrm{s}$ & $\circ$ & $\mathrm{N}$ & D & $\begin{array}{l}\text { Plantas de mantención y } \\
\text { desarrollo }\end{array}$ \\
\hline & & D & & & & & & Higgi & Bio & & & & & & \\
\hline & & $\mathrm{F}$ & $E$ & $F$ & M & A & $\mathrm{M}$ & ᄀ & 7 & $\mathrm{A}$ & $\mathrm{s}$ & 0 & $\mathrm{~N}$ & D & \\
\hline Tagasaste & Chamaecytisus & $\mathrm{c}$ & $E$ & $\mathrm{~F}$ & M & A & M & 2 & 2 & $\mathrm{~A}$ & $\mathrm{~s}$ & 0 & $\mathrm{~N}$ & D & desarrollo, plantas de producción \\
\hline & & D & & & & & & mbo & Arau & & & & & & de excedentes \\
\hline & $\begin{array}{l}\text { A. lanceolata, } A \text {. } \\
\text { petiolaris, } A \text {. }\end{array}$ & $\mathbf{F}$ & $E$ & $\mathrm{~F}$ & M & $\mathrm{A}$ & $\mathrm{M}$ & J & $\mathrm{J}$ & $\mathrm{A}$ & $\mathrm{s}$ & $\circ$ & $\mathrm{N}$ & D & \\
\hline $\begin{array}{c}\text { Corcolén } \\
\text { (Azara spo })\end{array}$ & integrifolia, A. & $\mathrm{c}$ & $E$ & $\mathrm{~F}$ & M & A & $\mathrm{M}$ & $\mathrm{d}$ & $\mathrm{u}$ & A & $\mathrm{s}$ & $\circ$ & $\mathrm{N}$ & D & $\begin{array}{l}\text { Plantas de estimulación, plantas } \\
\text { de mantención y desarrollo }\end{array}$ \\
\hline & $\begin{array}{l}\text { microphylia, } \\
\text { otras }\end{array}$ & D & & & & & & mbo & Arat & & & & & & \\
\hline & & $\mathbf{F}$ & $E$ & $\mathrm{~F}$ & M & A & $\mathrm{M}$ & $\begin{array}{ll} \\
\end{array}$ & 7 & $\mathrm{A}$ & $s$ & 0 & $\mathrm{~N}$ & D & Plantas de estimulación, plantas \\
\hline $\begin{array}{l}\text { Gomero } \\
\text { dulce }\end{array}$ & $\begin{array}{l}\text { Eucalyptus } \\
\text { cladocalyx }\end{array}$ & c & $E$ & $\mathrm{~F}$ & M & A & $\mathrm{M}$ & $\begin{array}{ll} \\
\end{array}$ & 7 & $\mathrm{A}$ & $s$ & 0 & $\mathrm{~N}$ & $\mathrm{D}$ & $\begin{array}{l}\text { de mantención y desarrollo, } \\
\text { plantas de producción de }\end{array}$ \\
\hline & & D & & & & & & cama & Higg & & & & & & excedentes \\
\hline & & $\mathbf{F}$ & $E$ & $\mathrm{~F}$ & M & A & $\mathrm{M}$ & $\mathrm{d}$ & $\mathrm{d}$ & A & $\mathrm{s}$ & 0 & $\mathrm{~N}$ & D & \\
\hline $\begin{array}{l}\text { Gomera o } \\
\text { Eucalipto rojo }\end{array}$ & $\begin{array}{c}\text { Eucalyptus } \\
\text { camaldutensis }\end{array}$ & $\mathrm{c}$ & $E$ & $\mathrm{~F}$ & M & A & $\mathrm{M}$ & $\mathrm{d}$ & $\mathrm{a}$ & A & $s$ & $\circ$ & $\mathrm{N}$ & $\mathrm{D}$ & desarrollo, plantas de producción \\
\hline & & D & & & & & & ma. & Arau & & & & & & de excedentes \\
\hline & & $\mathbf{F}$ & $\mathrm{E}$ & $\mathrm{F}$ & M & A & $\mathrm{M}$ & J & 7 & A & $\mathrm{s}$ & $\circ$ & $\mathrm{N}$ & D & \\
\hline Boj amarillo & $\begin{array}{l}\text { Eucalyotus } \\
\text { melliodora }\end{array}$ & $\mathrm{c}$ & $E$ & $\mathrm{~F}$ & M & A & M & J & a & A & $\mathrm{s}$ & $\circ$ & $\mathrm{N}$ & D & $\begin{array}{l}\text { Plantas de producción de } \\
\text { excedentes }\end{array}$ \\
\hline & & D & & & & & & ma. & Arau & & & & & & \\
\hline & & $\mathbf{F}$ & $\mathrm{E}$ & $\mathrm{F}$ & M & A & $\mathrm{M}$ & j & a & A & $\mathrm{s}$ & $\circ$ & $\mathrm{N}$ & D & \\
\hline Aromo pais & $\begin{array}{l}\text { Acacia } \\
\text { dealiatata }\end{array}$ & $\mathrm{c}$ & $E$ & $\mathrm{~F}$ & M & A & $\mathrm{M}$ & 2 & $\mathrm{~d}$ & A & $\mathrm{s}$ & 0 & $\mathrm{~N}$ & D & Plantas de estimulación \\
\hline & & D & & & & & & aiso & Arat & & & & & & \\
\hline & & $\mathbf{F}$ & $E$ & $\mathrm{~F}$ & M & A & M & J & 2 & A & $s$ & 0 & $\mathrm{~N}$ & D & \\
\hline Avellano chileno & $\begin{array}{l}\text { Gevuina } \\
\text { avellana }\end{array}$ & c & $E$ & $\mathrm{~F}$ & M & A & $\mathrm{M}$ & J & a & $\mathrm{A}$ & $s$ & 0 & $\mathrm{~N}$ & D & desarrollo, plantas de producción \\
\hline & & D & & & & & & imbo & lagal & & & & & & de excedentes \\
\hline & & $\mathbf{F}$ & $E$ & $\mathrm{~F}$ & M & A & M & $\mathrm{J}$ & $\mathrm{J}$ & $\mathrm{A}$ & $\mathrm{s}$ & 0 & $\mathrm{~N}$ & D & \\
\hline Tiaca & $\begin{array}{l}\text { Caldcluvia } \\
\text { paniculata }\end{array}$ & c & $E$ & $\mathrm{~F}$ & M & A & $\mathrm{M}$ & J & $\mathrm{u}$ & $\mathrm{A}$ & $\mathrm{s}$ & $\circ$ & $\mathrm{N}$ & D & $\begin{array}{l}\text { Plantas de producción de } \\
\text { excedentes }\end{array}$ \\
\hline & & D & & & & & & Bio- & galla & & & & & & \\
\hline & & $\mathrm{F}$ & $E$ & $\mathrm{~F}$ & M & A & $\mathrm{M}$ & $J$ & J & A & $s$ & 0 & $\mathrm{~N}$ & D & \\
\hline Tineo & $\begin{array}{l}\text { Weinmannia } \\
\text { trichosperma }\end{array}$ & $\mathrm{c}$ & $E$ & $F$ & M & A & $\mathrm{M}$ & ᄀ & $\mathrm{s}$ & $\mathrm{A}$ & $\mathrm{s}$ & $\circ$ & $\mathrm{N}$ & D & desarrollo, plantas de producción \\
\hline & & D & & & & & & Higg & Ays & & & & & & \\
\hline & & $F$ & $E$ & $F$ & $M$ & A & $\mathrm{M}$ & $\mathrm{J}$ & $\mathrm{J}$ & A & $s$ & 0 & $\mathrm{~N}$ & D & \\
\hline Pelú & $\begin{array}{l}\text { Sophora } \\
\text { microphyla }\end{array}$ & $\mathrm{c}$ & $E$ & $\mathrm{~F}$ & M & $\mathrm{A}$ & $\mathrm{M}$ & J & 7 & $\mathrm{A}$ & $\mathrm{s}$ & $\circ$ & $\mathrm{N}$ & $\mathrm{D}$ & Plantas de estimulación \\
\hline & & $\mathrm{D}$ & & & & & & Maul & ysén & & & & & & \\
\hline & & $F$ & $E$ & $F$ & $M$ & A & $\mathrm{M}$ & $\mathrm{J}$ & $\mathrm{J}$ & $\mathrm{A}$ & $s$ & 0 & $\mathrm{~N}$ & $D$ & \\
\hline Notro o Ciruelillo & $\begin{array}{l}\text { Embotrium } \\
\text { coccineum }\end{array}$ & $\mathrm{c}$ & $E$ & $\mathrm{~F}$ & M & A & M & J & J & A & $s$ & $\circ$ & $\mathrm{N}$ & D & $\begin{array}{l}\text { Plantas de estimulación, plantas } \\
\text { de mantención y desarrollo }\end{array}$ \\
\hline & & D & & & & & & ule - & gallar & & & & & & \\
\hline
\end{tabular}


El calendario del Cuadro $\mathrm{N}^{\circ} 2$ permite tener una idea general del periodo anual de disponibilidad de flores y de la función de esas especies dentro del ciclo biológico de las abejas. Cabe hacer notar que las especies que florecen en la época invernal tienen una función de estimulación, es decir, generan que la abeja comience a salir de la colmena a pecorear las flores, de modo de dar inicio a la producción de miel, como ejemplo están boldo, pelú y aromo país.

Las especies cuya función está definida como de producción de excedente son relevantes para el negocio apícola porque ofrecen un nivel de néctar que va más allá de lo que la colmena necesita, en este caso se dispondrá de mayor cantidad de miel que puede ser cosechada por el apicultor, en este grupo están ulmo, tiaca y la mayoría de los eucaliptos, entre otras.

El calendario también es útil para programar la cosecha de semillas de las especies de interés, según los meses del año. Por ejemplo durante el mes de enero se puede cosechar la semilla de 11 de las 23 especies del cuadro anterior, entre las cuales se encuentran pelú, tineo, y avellano, entre otras.

Este calendario podrá requerir ajustes antes de usarse en áreas ambientalmente muy distintas, pues en zonas más cálidas la floración se produce antes, además en un escenario climático cambiante puede haber importantes variaciones de un año a otro. Esta herramienta también puede ser muy útil para el desarrollo de un sistema de manejo de colmenas que tienda a la producción de mieles diferenciadas, extrayéndose la miel producida al finalizar el periodo de floración de alguna especie en particular o de un conjunto de ellas.

A partir de la información anterior se inició la localización de las especies seleccionadas, la cosecha de semillas y la revisión de antecedentes de tratamientos germinativos y protocolos de viverización para la producción de las plantas requeridas. Hasta ahora se ha producido un total de 33.700 plantas aproximadamente en el vivero institucional de INFOR Bio Bio (Cuadro $N^{\circ} 3$ ).

Como aprecia en el Cuadro $\mathrm{N}^{\circ} 3$, existe variabilidad en la cantidad de plantas producidas de cada especie. Esto obedece a diversas razones, como escasa semilla, baja viabilidad, baja germinación por falta de protocolos o pretratamientos conocidos y otras. Esto sugiere la necesidad de monitoreo por más de una temporada y de realizar ensayos para definir los protocolos, tanto de producción de plantas como de control de algunas plagas.

Los huertos se establecieron con especies adaptadas a las condiciones locales de clima y suelo del lugar en cada caso, consiguiéndose una matriz de 13 especies combinadas en las distintas unidades.

Durante la temporada 2015 y 2016 se instalaron 7 y 26 huertos, respectivamente, lo que constituye un total de 33 huertos que ocupan alrededor de 30 ha efectivas. En el Cuadro $N^{\circ} 4$ se presenta el número y superficie de huertos por provincia y comuna.

Como se observa en el Cuadro $\mathrm{N}^{\circ} 4$, se establecieron huertos desde menos de media hectárea y hasta de dos hectáreas. La Agricultura Familiar Campesina de la región desarrolla principalmente una agricultura de autoconsumo y la apicultura en general es una actividad que le genera ingresos adicionales. Así, los terrenos disponibles para la plantación de árboles son más bien escasos.

El establecimiento de las unidades melíferas debía constituirse en un elemento complementario en la propiedad y no alterar el uso agrícola habitual que le da el propietario. En algunos casos los árboles fueron establecidos en el perímetro de las propiedades o en pequeños sectores sin uso agrícola.

Esta situación agregó complejidad a la operación del proyecto y un aumento de costos, dado que se había planificado establecer huertos de 4 hectáreas. El elemento positivo de esta situación fue que es posible impactar a un número mucho mayor de apicultores que no tenían la posibilidad, por sus medios, de establecer estas unidades melíferas. 


\section{Cuadro $\mathrm{N}^{\circ} 3$ \\ PLANTAS PRODUCIDAS TEMPORADAS 2015-2016}

\begin{tabular}{|c|c|c|c|}
\hline Especie & Nombre Científico & Pretratamiento de Semillas & $\begin{array}{l}\text { Plantas } \\
\left(\mathbf{N}^{\circ}\right)\end{array}$ \\
\hline Bollén & Kageneckia oblonga & Remojo con Ac. Giberélico 24 h. & 978 \\
\hline Robinia & Robinia pseudoacacia & $\begin{array}{l}\text { Remojo en agua recién hervida y dejar enfriar } \\
\text { por } 24 \text { h. }\end{array}$ & 2.466 \\
\hline Guindo Santo & Eucryphia glutinosa & Remojo con Ac. Giberélico 24 h. & 2.951 \\
\hline Pelú & Sophora cassioides & Remojo con Ac. Giberélico 24 h. & 744 \\
\hline Boldo & Peumus boldus & $\begin{array}{l}\text { Estratificación fría por } 30 \text { días y después } 24 \text { h } \\
\text { remojo en Ac. Giberélico }\end{array}$ & 645 \\
\hline Arrayán & Luma apiculata & Remojo con Ac. Giberélico 24 h. & 625 \\
\hline Canelo & Drimys winteri & Remojo con Ac. Giberélico 24 h. & 1.731 \\
\hline Quillay & Quillaja saponaria & Remojo en agua a temperatura ambiente $24 \mathrm{~h}$. & 4.114 \\
\hline Chagual & Puya chilensis & $\mathrm{S} / \mathrm{l}$ & 24 \\
\hline Ciprés de la Cordillera & Austrocedrus chilensis & Remojo con Ac. Giberélico 24 h. & 57 \\
\hline Huingán & Schinus polygamus & Remojo con Ac. Giberélico 24 h. & 12 \\
\hline Maqui & Aristotelia chilensis & Remojo con Ac. Giberélico 24 h. & 1.271 \\
\hline Avellano & Gevuina avellana & Remojo en agua a temperatura ambiente $48 \mathrm{~h}$. & 421 \\
\hline Maitén & Maytenus boaria & Remojo con Ac. Giberélico 24 h. & 2.020 \\
\hline Peumo & Cryptocarya alba & Sin tratamiento & 1.746 \\
\hline Notro & Embothrium coccineum & Remojo con Ac. Giberélico 24 h. & 1.132 \\
\hline Murta & Ugni molinae & Propagación por estacas & 276 \\
\hline Mardroño & Escallonia pulverulenta & Remojo con Ac. Giberélico 24 h. & 4.517 \\
\hline Madroño & Arbutus unedo & Remojo con Ac. Giberélico 24 h. & 756 \\
\hline Avellanillo & Lomatia dentata & Remojo con Ac. Giberélico 24 h. & 436 \\
\hline Radal & Lomatia hirsuta & Remojo con Ac. Giberélico 24 h. & 299 \\
\hline Patagua & Crinodendron patagua & Remojo con Ac. Giberélico 24 h. & 528 \\
\hline Corcolén & Azara serrata & $\begin{array}{l}\text { Remojo con Ac. Giberélico } 24 \text { h. y } \\
\text { enraizamiento de estacas }\end{array}$ & 775 \\
\hline Laurel & Laurelia sempervirens & Siembra directa en almácigo & 469 \\
\hline Ulmo & Eucryphia cordifolia & Remojo con Ac. Giberélico 24 h. & 997 \\
\hline Tuja & Thuja sp & Remojo con Ac. Giberélico 24 h. & 484 \\
\hline Gomero Coral & Eucalyptus torquata & Remojo en agua a temperatura ambiente $24 \mathrm{~h}$. & 59 \\
\hline Boj Amarillo & Eucalyptus melliodora & Remojo con Ac. Giberélico 24 h. & 677 \\
\hline Gomero Sidra & Eucalyptus gunnii * & Remojo con Ac. Giberélico 24 h. & 67 \\
\hline Gomero Rojo & Eucalyptus camaldulensis & Remojo con Ac. Giberélico 24 h. & 922 \\
\hline Gomero Dulce & Eucalyptus cladocalyx & Remojo con Ac. Giberélico 24 h. & 254 \\
\hline Gomero Limón & Corymbia citriodora & Remojo con Ac. Giberélico 24 h. & 1.278 \\
\hline \multicolumn{3}{|l|}{ Total } & 33.731 \\
\hline
\end{tabular}


Cuadro $\mathrm{N}^{\circ} 4$

NÚMERO Y SUPERFICIE DE HUERTOS ESTABLECIDOS EN LA REGIÓN DEL BIO BIO TEMPORADA 2015-2016

\begin{tabular}{|c|r|r|}
\hline Provincia / Comuna & $\begin{array}{c}\text { Huertos } \\
\left(\mathbf{N}^{\circ} \mathbf{)}\right.\end{array}$ & $\begin{array}{c}\text { Superficie } \\
\text { (ha) }\end{array}$ \\
\hline Arauco & 3 & 2 \\
\hline Arauco & 3 & 2 \\
\hline Biobío & 3 & 4 \\
\hline Mulchén & 1 & 2 \\
\hline Negrete & 1 & 1 \\
\hline Yumbel & 1 & 1 \\
\hline Concepción & 6 & 6 \\
\hline Florida & 3 & 4 \\
\hline Hualqui & 3 & 2 \\
\hline Bulnes & 21 & 17,5 \\
\hline Nuble & 3 & 2,5 \\
\hline El Carmen & 1 & 0,5 \\
\hline Pinto & 2 & 1,5 \\
\hline Portezuelo & 1 & 0,5 \\
\hline Quillón & 1 & 0,5 \\
\hline Quirihue & 8 & 7,6 \\
\hline Ranquil & 2 & 1,5 \\
\hline San Carlos & 1 & 0,4 \\
\hline San Nicolás & 1 & 0,5 \\
\hline Total Unidades & 1 & 2 \\
\hline & 33 & 29,5 \\
\hline
\end{tabular}

Un aspecto adicional interesante es que la dispersión de los apicultores permite conectar la distribución de importantes especies forestales que actualmente presenta una distribución fragmentada. Esto representa un elemento de interés para los apicultores cuando reconocen que su labor puede contribuir a la recuperación y conservación del bosque nativo o bien a la protección de especies vulnerables.

Para la mantención de los huertos establecidos en la temporada 2015 se definió un programa de riegos durante la época estival, gracias a esto la supervivencia promedio fue superior el $90 \%$. Durante la temporada 2016, conjuntamente con establecer los restantes huertos se procedió a reponer las plantas muertas de la temporada anterior.

El proceso de mantención se ha visto facilitado por la cooperación y conocimiento de los apicultores. Ellos revisan las plantas de sus huertos y si notan alguna alteración en el follaje o el vigor de la planta informan a INFOR, en algunos casos enviando fotografías. En la medida que aumenta el número de huertos este procedimiento se transforma en indispensable para controlar los daños en estos.

En relación al riego, en la mayoría de los casos es manual por lo que a través de instrumentos de otros servicios del agro, como INDAP y CNR, se están presentando proyectos para cada uno de los apicultores que les permitan instalar riego por goteo. Este es otro elemento de gestión que debe utilizarse en este tipo de programas dirigidos a la agricultura familiar campesina (AFC). 


\section{Capacitación en Producción de Flores para la Apicultura}

Durante el desarrollo del programa hasta ahora se han realizado distintas capacitaciones:

Cursos de Injertación y Producción de Plantas con Utilidad Melífera: Dos cursos con una participación aproximada de 30 apicultores en cada uno. Objetivo principal es capacitar monitores. Existe un número importante de apicultores que reproducen plantas, especialmente agrícolas, y denotaron gran interés en el tema de plantas para fines melíferos.

Complementariamente, INFOR puso sus instalaciones a disposición de tales apicultores, para que hiciesen pasantías en los momentos claves del proceso de producción de plantas, siembra, repique, control sanitario, endurecimiento, entre otros, no obstante no hubo concurrencia.

Será necesario desarrollar otras instancias de aprendizaje dado que la lejanía de los apicultores y los recursos de que ellos disponen para movilizarse son factores muy limitantes.

Ambos cursos fueron desarrollados por especialistas de INFOR en los temas de elementos y manejo de viveros, producción de plantas por semilla, plantas producidas por propagación vegetativa e injertación. Todos los asistentes recibieron un diploma formal por su participación a cada uno de los cursos.

Plantaciones de Especies Nativas Melíferas: Esta capacitación se abordó directamente con las plantaciones de huertos en la temporada 2016. Se realizaron 3 capacitaciones en la región del Bio Bio y una en la región de La Araucanía. Las primeras en las comunas de Chillán Viejo, El Carmen, Negrete y Mulchén, y la última en la comuna de Los Sauces.

En las capacitaciones de la región de Bio Bio asistieron entre 10 a 15 personas y en la de La Araucanía un número cercano a 30 . En todos los casos, se entregaron los antecedentes la capacitación, la razón de los elementos de esta y la forma en que se realizaría la labor, y luego se procedió a hacer una demostración práctica. En seguida, los apicultores presentes realizaron la tarea siguiendo las instrucciones.

Al finalizar la jornada, se les entregaron de regalo plantas melíferas a los apicultores para que las establecieran en su propiedad y en algunos casos se les proporcionó el fertilizante y el gel requerido.

Parámetros de Calidad de Miel y Cera de Abejas y su Importancia en la Comercialización: A este curso asistieron 27 personas. Algunos de ellos eran apicultores que habían puesto a disposición sus mieles de la temporada 2015-2016 para análisis Físico-Químico y Melisopalinológico.

El curso desarrolló los temas de calidad de miel y análisis de resultados físicos y químicos de las mismas; Revisión del Reglamento Sanitario de los Alimentos; Codex Alimentarius y Etiquetado Nutricional; y Principios de la evaluación sensorial de mieles, Calidad de la cera y su importancia en la producción del colmenar y Revisión de la Norma Chilena - "Cera de abejas - Requisitos".

Posteriormente, se realizó un taller donde se aplicó un Test de Sensibilidad de los Cuatro Sabores a cada asistente: Nivel de azúcar, nivel de sal, nivel de ácido y nivel de amargo, para entre ellos identificar posibles relatores para la formación de un grupo que trabaje en la denominación de origen de las mieles regionales. Por último, se degustaron las mieles del grupo con el objeto de describirlas de acuerdo a la pauta de familias y subfamilias de olores y aromas de la miel. 


\section{Caracterización Preliminar de la Miel Regional}

Se analizó un total de 61 muestras de miel, cosechadas en la región del Bio Bio durante la temporada 2016, a las que se les evaluaron los parámetros físico-químicos relevantes para su comercialización. En el Cuadro $\mathrm{N}^{\circ} 5$ se resumen los estándares que debe cumplir la miel para que pueda ser comercializada para consumo humano.

\section{Cuadro $\mathrm{N}^{\circ} 5$ \\ REQUISITOS FÍSICO-QUÍMICOS DE LA MIEL COMERCIALIZADA PARA CONSUMO HUMANO SEGÚN LA NORMA CHILENA (NCh616) Y SU RESPECTIVO MÉTODO DE ENSAYO}

\begin{tabular}{|c|c|c|}
\hline Requisitos & Miel de Flores & $\begin{array}{c}\text { Método de Ensayo } \\
\text { según Norma }\end{array}$ \\
\hline Sólidos insolubles en agua (\%) & $\begin{array}{c}\leq 0,1 \\
\text { Miel Prensada } \\
\leq 0,5\end{array}$ & NCh 3047 \\
\hline Contenido de Agua (\%) & $\leq 20$ & NCh 3026 \\
\hline Acidez libre (meq / kg) & $\leq 50$ & NCh 3019 \\
\hline Fructuosa y glucosa (como suma de ambas) (\%) & $\geq 60$ & NCh 574 \\
\hline Sacarosa (\%) & $\leq 5$ & NCh 574 \\
\hline Cenizas (\%) & $\leq 0,6$ & NCh 3002 \\
\hline Conductividad electrica $(\mathrm{mS} / \mathrm{cm})$ & $\leq 0,8$ & NCh 3064 \\
\hline Hidroximetilfurfural (HMF) (mg / kg) & $\begin{array}{l}\qquad \leq 40 \\
\text { Condicionado a mieles con un contenido bajo de enzimas } \\
\text { naturales } \\
\text { Ej. Mieles de cítricos } \leq 15 \text { Miel tropical } \leq 80\end{array}$ & NCh 3046 \\
\hline Índice de diastasa (escala Shade) & $\begin{array}{l}\text { Determinado después de la elbaroración o mezcla } \geq 8 \\
\text { Mieles con un contenido bajo de enzimas naturales (Ej. } \\
\text { Mieles de cítricos) y un contenido bajo de HMF (no mayor } \\
\text { que } 15 \mathrm{mg} / \mathrm{kg} \text { ) } \geq 3\end{array}$ & NCh 3087 \\
\hline Determinación de metales pesados $\mathrm{Cu}, \mathrm{Cr}, \mathrm{Cd}, \mathrm{Pb}$ & $\begin{array}{l}\text { Libre de metales pesados que puedan ocasionar un daño a la } \\
\text { salud humana. Niveles máximos establecidos por CODEX } \\
\text { Alimentarius Commission o lo establecido por la autoridad } \\
\text { competente. }\end{array}$ & NCh 3142 \\
\hline
\end{tabular}

A continuación se presentan y analizan los resultados de la caracterización de las mieles de la región del Bio Bio en función de las cinco categorías de parámetros descritos en la metodología.

\section{- Parámetros Relacionados con la Madurez o Sazón de la Miel. Humedad}

El Contenido de Humedad es un valor crítico para la comercialización de la miel y se relaciona con su madurez al momento de la cosecha. Para una miel madura se señala que no debe exceder del $18 \%$ de humedad, de lo contrario es muy probable que se produzca fermentación y se alteren el color, sabor y viscosidad. Sin embargo, como se observa en el Cuadro $N^{\circ} 5$, la Norma Chilena acepta valores de contenido de humedad de hasta $20 \%$. Los azúcares constitutivos de la miel se transforman, aumenta la acidez, se pierden las actividades enzimáticas y las actividades biológicas (antibacterianas). La cercanía a este valor es indicativa también del momento oportuno de la cosecha. Por otra parte, el bajo contenido de humedad, le confiere un alto poder hidrofílico, por lo que será capaz de absorber agua fácilmente. Dentro de los azúcares la fructosa es más higroscópica que la glucosa, por ello, cuando se hidrata la miel o absorbe humedad de la atmósfera o por el propio proceso de envejecimiento, se puede producir una separación de fases, quedando la glucosa en el fondo, y la fructosa en una solución más acuosa en la parte superior, aumentando así el peligro de fermentación. 
En la Figura $\mathrm{N}^{\circ} 1$ se muestra el contenido de humedad de las 61 muestras evaluadas de mieles de Bio Bio, en ella se observa que los valores fluctuaron entre 14,70 y 19,20\%, por ende todos dentro de la norma chilena.

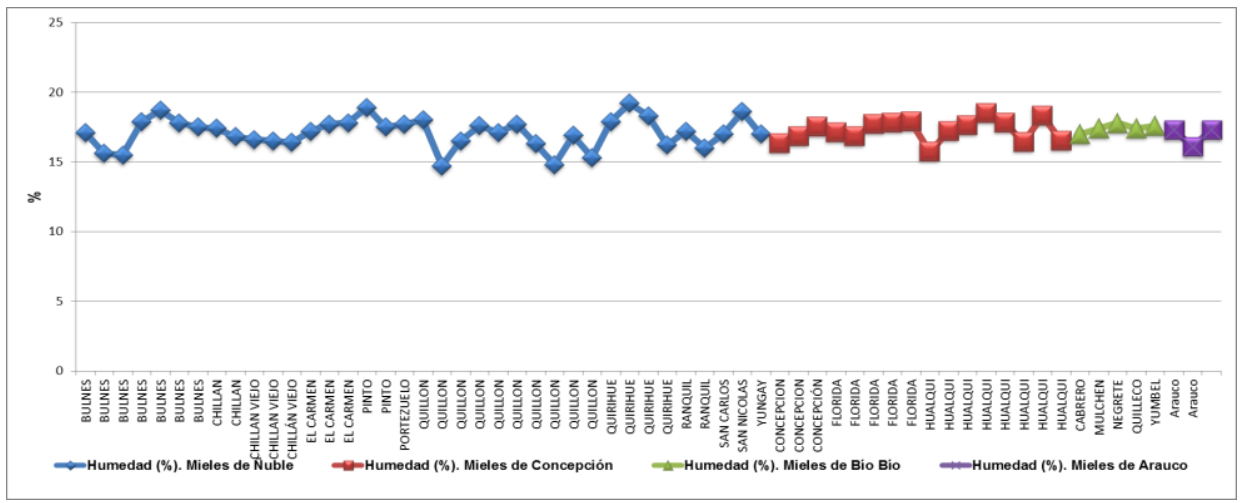

\section{Figura $\mathbf{N}^{\circ} 1$ \\ CONTENIDO DE HUMEDAD PARA MIELES POR PROVINCIAS Y COMUNAS}

\section{Parámetros Relacionados con el Manejo Posterior a la Cosecha. Hidroximetil furfural (HMF)}

Este parámetro se relaciona con el manejo de la miel. Cuando esta se expone a temperaturas superiores a $40^{\circ} \mathrm{C}$ se descompone la fructosa. $\mathrm{A}$ pH bajos también se relaciona con la aparición de HMF. Valores altos puede indicar que el apiario se encuentra directo al sol.

Como se observa en la Figura $\mathrm{N}^{\circ} 2$, en las muestras analizadas el valor fluctuó entre 0,0 y $11,90 \mathrm{mg} / \mathrm{kg}$, por lo cual todas las mieles analizadas cumplen la Norma señaladas en el Cuadro $\mathrm{N}^{\circ} 5$.

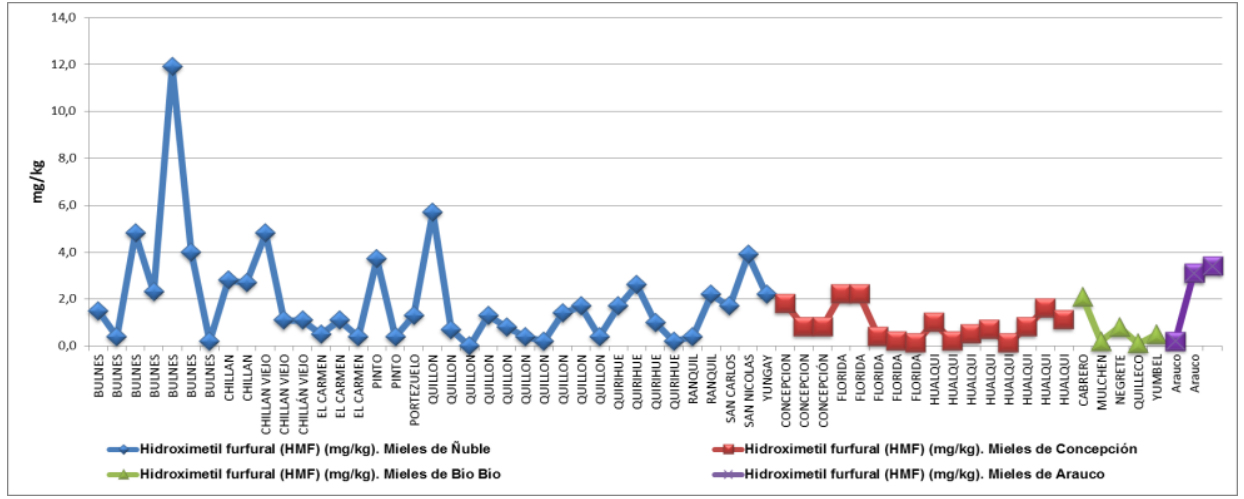

Figura $\mathrm{N}^{\circ} 2$

HIDROXIMETIL FURFURAL (HMF) POR PROVINCIAS Y COMUNAS 


\section{- Parámetros Relacionados con la Caracterización de la Miel. Ceniza}

Corresponden a un indicador del contenido de minerales, la importancia de estos en la caracterización del origen geográfico de la miel se debe a que aquellos presentes en la miel dependerán del tipo de suelo en que crecen las plantas que pecorean las abejas, de modo que una miel que proviene de una especie floral puede ser distinta si esta especie está situada en distintas región geográfica, donde los suelos tengan distinta composición o contenido de minerales. En las muestras evaluadas el contenido de cenizas varió entre 0,08 y $1,14 \%$.

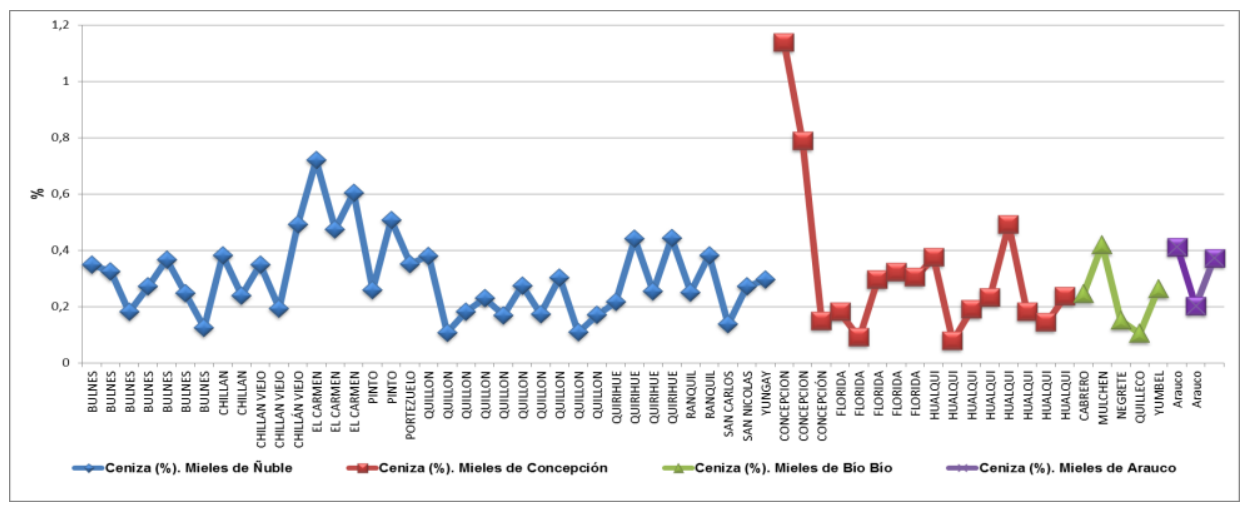

Figura $\mathbf{N}^{\circ} 3$

CENIZAS PRESENTES EN LAS MIELES MUESTREADAS POR PROVINCIA Y COMUNAS

Según la Norma Chilena NCh616 el contenido de cenizas en la miel para consumo humano debe ser inferior a 0,6\%. Como se aprecia en la Figura $N^{\circ} 3$ existen 3 muestras de mieles, 2 de la Comuna de Concepción y una de la Comuna de El Carmen, que superan este valor.

Algunos autores señalan que este es un límite muy discutible, puesto que en las mieles oscuras, el contenido puede ser superior, sin que esto se necesariamente indeseable, ya que representaría un aporte de sales minerales importantes para la alimentación humana (Cornejo, 1993, citado por Díaz, 2003).

\section{Parámetros Relacionados con la Frescura de la Miel. Índice de Diastasa}

Este índice es un indicador del nivel de frescura, donde valores muy bajos (inferiores a 0,8 en escala de Shade) indican recalentamiento y/o envejecimiento de la miel.

En las muestras analizadas se observó una alta variabilidad para este parámetro $(7,10$ a 86,70 en escala de Shade), no obstante, solo una muestra no cumple con el valor mínimo de 0,8 establecido en la norma pertinente. En esta muestra se debe revisar el contenido de HMF, pues si este es inferior a $15 \mathrm{mg} / \mathrm{kg}$, entonces el valor umbral de diastasa exigido por la norma se reduce a 3 en escala de Shade, caso en el que la muestra cuestionada estaría cumpliendo el requisito para diastasa exigido en la norma para mieles con bajo HMF. 


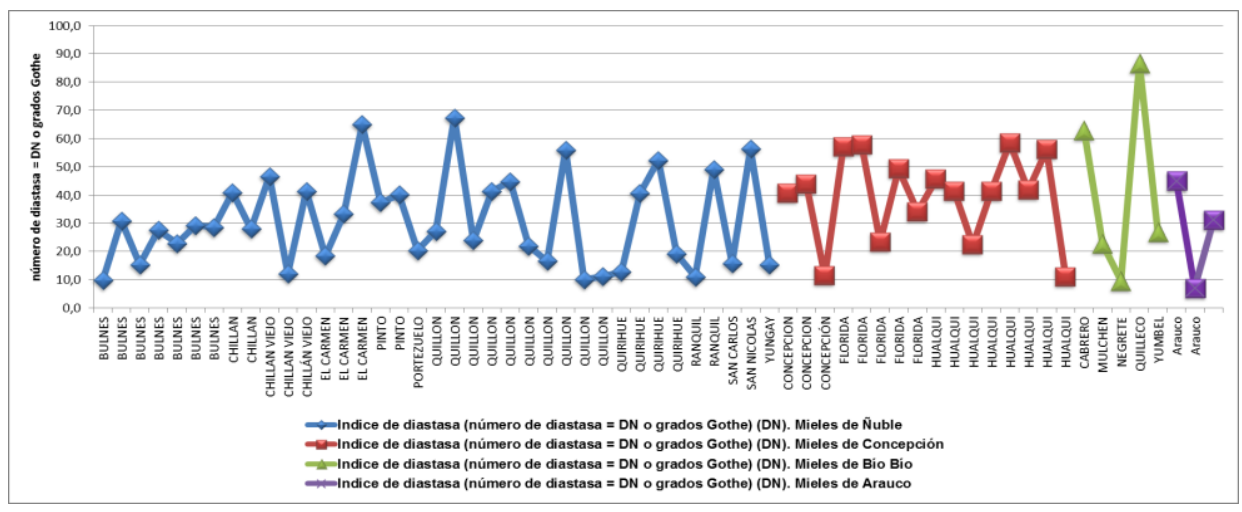

Figura $N^{\circ} 4$

ÍNDICE DE DIASTASA POR PROVINCIAS Y COMUNAS

\section{Parámetros Relacionados con el Grado de Antioxidantes de la Miel. Índice del Contenido de Compuestos Antioxidantes Filtradores de la Luz UV}

Los antioxidantes filtradores de la luz UV pueden ser interesantes desde el punto de vista de la industria cosmética (cremas, labiales lociones, otros). Para este parámetro se prefieren valores altos. En las muestras el valor fluctuó entre 13 y $1.029 \mathrm{mg}$ de ácido gálico por kg de miel.

Los valores mayores se encuentran en mieles provenientes de las comunas de Hualqui, Florida y Quillón, sin embargo, en general existe alta variabilidad a nivel provincial. No existen valores que estén señalados en la Norma chilena.

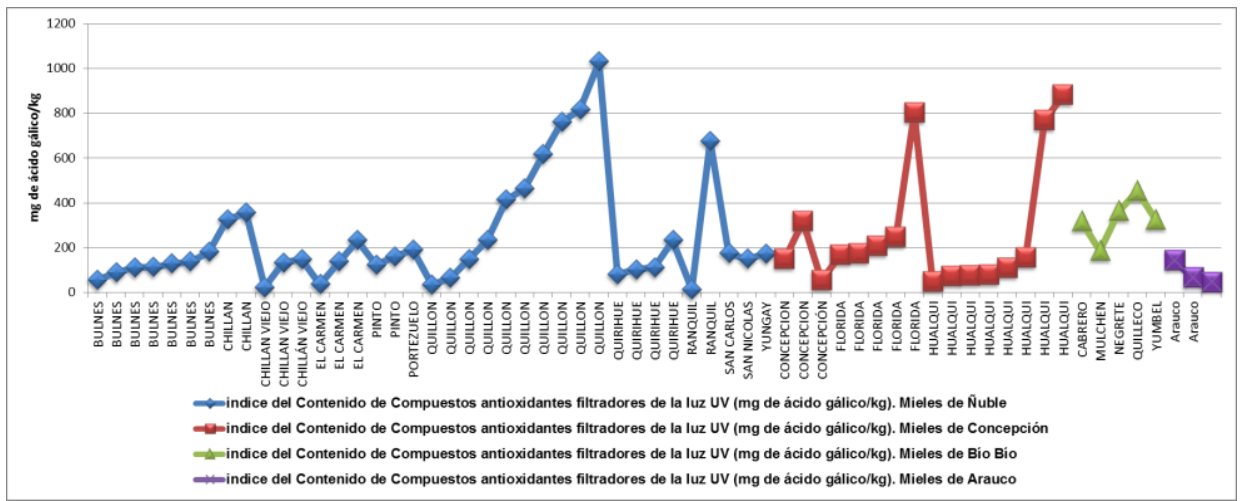

\section{Figura $N^{\circ} 5$}

ÍNDICE DE COMPUESTOS ANTIOXIDANTES FILTRADORES DE LA LUZ UV PARA MIELES POR PROVINCIAS Y COMUNAS

La información que se genera con la evaluación de los parámetros físico químicos de la miel puede contribuir a la toma de decisiones relacionadas con la selección de especies y el manejo posterior de los huertos melíferos tendiente a la producción de mieles diferenciadas. En este sentido el proyecto también contempla el inicio del proceso de obtención de una denominación 
de origen de la miel que se produzca en la región claramente identificada en su calidad bioquímica y botánica.

\section{APROXIMACIÓN A LA APICULTURA PATAGÓNICA}

La apicultura en la Patagonia constituye un gran desafío debido a condiciones climáticas muy adversas para esta actividad al sur del estuario de Reloncaví. La búsqueda de alternativas productivas, pensando en una producción con buena aceptación en el mercado y el aprovechamiento de ventajas comparativas, como la imagen patagónica y la escasa contaminación ambiental, sugiere realizar investigación para evaluar la factibilidad de la producción melífera en estas extensas regiones.

Los aspectos de clima podrían se contrarrestados con selección y mejoramiento genético, sanidad y nutrición de las abejas y un ajuste minucioso de un paquete tecnológico adhoc. Trabajos efectuados en Argentina permiten afirmar que la producción melífera sería factible, optimizando al máximo todas las actividades del proceso productivo (Scatturice et al., 2005).

En la región patagónica chilena, las cifras censales disponibles (INE, 2017) señalan que en la región de Aysén habría 4 apicultores y que en la región de Magallanes la apicultura sería una actividad inexistente.

En el caso de la región patagónica argentina, la actividad apícola se localiza mayoritariamente en los valles irrigados y presenta un fuerte crecimiento. Para el Censo Nacional Agropecuario 1988 en ese país (Instituto Nacional de Estadística y Censos, 1990) existían 989 establecimientos agropecuarios con colmenas en las provincias de Neuquén, Río Negro, Chubut y Santa Cruz, con un total de 19.503 colmenas, llegando en 2005 a más que duplicar esta cantidad (245\%), como resultado de un fuerte proceso de crecimiento de la actividad, que sigue en la misma tendencia pese al nivel de los precios.

La región patagónica argentina ha exportado miel, durante el año 2004, por más de US \$ 1.000 .000 y puede incrementar este monto mediante el aseguramiento de la calidad (por ejemplo la miel libre de residuos) y la denominación de origen.

Cuadro $\mathrm{N}^{\circ} 6$

EVOLUCIÓN DE LA POBLACIÓN DE COLMENAS PATAGONIA ARGENTINA

\begin{tabular}{|l|r|r|r|r|}
\hline \multirow{2}{*}{ Provincia } & $\mathbf{1 9 3 7}$ & $\mathbf{1 9 8 8}$ & $\mathbf{2 0 0 3}$ & $\mathbf{2 0 0 5}$ \\
\cline { 2 - 5 } & \multicolumn{4}{|c|}{$\left(\mathbf{N}^{\circ}\right)$} \\
\hline Neuquén & 2.241 & 2.143 & 12.241 & 15.407 \\
\hline Río Negro & 19.609 & 15.789 & 21.500 & 34.687 \\
\hline Chubut & 2.551 & 1.316 & 1.598 & 3.000 \\
\hline Santa Cruz & 18 & 255 & 300 & 500 \\
\hline Total & 21.850 & 19.503 & 35.339 & 50.394 \\
\hline
\end{tabular}

(Fuente: INTA, 2016)

De acuerdo con Massaccesi (2002) en la Patagonia Argentina la apicultura es una actividad tradicional orientada a satisfacer el autoconsumo de miel. Por eso el perfil de los apicultores es de una reducida cantidad de colmenas, con un buen seguimiento de las mismas a lo largo de la temporada y un trabajo verdaderamente artesanal. Otra ventaja interesante es que existe un buen hábito de consumo de productos apícolas. 
Además del alto consumo de miel, el público en general tiene también una marcada preferencia por subproductos tales como polen, propóleos y jalea real producidos en la zona. Más allá de la importancia económica que esto pueda llegar a tener, está el hecho de mejorar ostensiblemente la calidad de vida de la gente.

Por razones climáticas pareciera que la temporada de trabajo de las abejas es relativamente corta en la región patagónica, pero presenta una serie de ventajas comparativas:

Las colonias invernan en forma efectiva. Con lo que se disminuye el consumo de miel y se produce un corte en el desarrollo de las colmenas durante el invierno.

Hay una sucesión de floraciones desde mediados de agosto hasta entrado el otoño que facilitan la evolución de la abeja y permiten cosechas escalonadas a lo largo de la primavera y el verano.

A diferencia de otras regiones productivas, los apicultores no poseen el hábito de uso indiscriminado de productos químicos (acaricidas, antibióticos, otros), gracias a lo cual las colmenas generan una resistencia natural contra las diversas enfermedades y paralelamente los productos apícolas prácticamente no presentan contaminaciones.

Si bien los factores climáticos juegan un papel relevante para esta actividad, existen otros países, con condiciones climáticas similares a las patagónicas, donde se han realizado ciertas adaptaciones. Por ejemplo, en el caso de Finlandia se ha desarrollado fuertemente la apicultura urbana, conllevando también la inclusión de un sector más joven en esta actividad (Manríquez, 2015).

Por otra parte, existen colmenas especialmente diseñadas para estos climas. Están fabricadas en poliestireno expandido (PSE) extra endurecido, de grado alimentario, con una densidad superior a los $100 \mathrm{~kg} / \mathrm{m}^{3}$. Gracias a su material, destacan por su resistencia, ligereza y una vida útil de muchos años.

Este tipo de colmenas ha sido desarrollado en Finlandia por Paradise Honey y el sistema ha sido probado bajo las exigentes condiciones climáticas (como en Siberia) a las que está expuesta la apicultura finlandesa. Los inviernos árticos, con temperaturas inferiores a $-35^{\circ} \mathrm{C}$, y los calurosos veranos, con marcas térmicas que superan los $30^{\circ} \mathrm{C}$, la hacen un colmena muy resistente a los diferentes ambientes y condiciones meteorológicas que se puedan presentar en todos los climas.

En términos generales las temperaturas externas afectan a las abejas, por debajo de los $10^{\circ} \mathrm{C}$ no hay actividad fuera de la colmena y a los $0^{\circ} \mathrm{C}$ la colmena entra en una especie de letargo, casi sin movimiento y actividad ni consumo de reservas, y puede resistir temperaturas muy bajas.

La reina para aparearse sale al exterior, requiere una temperatura óptima de 18 a $20^{\circ} \mathrm{C}, \mathrm{y}$ para iniciar una postura continua de huevos son necesarias temperaturas cercanas a los $30^{\circ} \mathrm{C}$. Las obreras, por su parte, empiezan a trabajar con una temperatura de unos $25^{\circ} \mathrm{C}$.

Para países más fríos, como Finlandia, Islandia, Suecia, estas son temperaturas que limitan bastante la producción de miel o la posibilidad de aumentar significativamente las colmenas año a año. Lo positivo de estos climas es que el desarrollo agrícola no enfrenta enfermedades y los monocultivos y los pesticidas no son una amenaza para mantener abejas vivas y sanas (Manríquez, A, 2015a).

La solución puede venir de generar condiciones ambientales adecuadas en etapas críticas de los ciclos biológicos de las abejas para la producción de miel y su reproducción.

En relación a las especies forestales o leñosas factibles de utilizar en la Patagonia, se describe una primera aproximación en el Cuadro $\mathrm{N}^{\circ} 7$. 
Cuadro $\mathrm{N}^{\circ} 7$

ESPECIES MELÍFERAS PRESENTES EN LA PATAGONIA, ÉPOCAS DE FLORACIÓN Y DE PRODUCCIÓN DE SEMILLAS, DISTRIBUCIÓN Y CARACTERÍSTICAS FENOLÓGICAS Y DE HÁBITO

\begin{tabular}{|c|c|c|c|c|c|c|c|c|c|c|c|c|c|}
\hline $\begin{array}{l}\text { Nombre } \\
\text { Común }\end{array}$ & $\begin{array}{l}\text { Nombre } \\
\text { Científico }\end{array}$ & \multicolumn{11}{|c|}{ CALENDARIZACIÓN Y DISTRIBUCIÓN GEOGRÁFICA } & Fenología y Hábito \\
\hline \multirow{3}{*}{ Michay } & \multirow{3}{*}{$\begin{array}{l}\text { Berberis } \\
\text { darwinii }\end{array}$} & $\mathbf{F}$ & & $\mathrm{F}$ & M & & & & $\mathrm{s}$ & 0 & $\mathrm{~N}$ & & \multirow{3}{*}{$\begin{array}{l}\text { Monoico } \\
\text { Siempreverde }\end{array}$} \\
\hline & & c & $\mathrm{E}$ & $\mathrm{F}$ & M & & & & & & & D & \\
\hline & & D & \multicolumn{10}{|c|}{ Maule - Aysén } & \\
\hline \multirow{3}{*}{ Zarcilla } & \multirow{3}{*}{$\begin{array}{l}\text { Berberis } \\
\text { empetrifolia }\end{array}$} & $\mathbf{F}$ & $E$ & & & & & & & & $\mathrm{~N}$ & D & \multirow{3}{*}{$\begin{array}{l}\text { Monoico } \\
\text { Siempreverde }\end{array}$} \\
\hline & & C & & $\mathrm{F}$ & M & & & & & & & & \\
\hline & & D & \multicolumn{10}{|c|}{ Coquimbo-Magallanes } & \\
\hline \multirow{3}{*}{ Calafate } & \multirow{3}{*}{$\begin{array}{l}\text { Berberis } \\
\text { microphylla } \\
\text { (o buxifolia) }\end{array}$} & $\mathbf{F}$ & $E$ & $\mathrm{~F}$ & M & & & A & $\mathrm{S}$ & 0 & $\mathrm{~N}$ & D & \multirow{3}{*}{$\begin{array}{l}\text { Monoico } \\
\text { Siempreverde }\end{array}$} \\
\hline & & C & & $\mathrm{F}$ & M & & & & & & & & \\
\hline & & D & \multicolumn{10}{|c|}{ Maule - Magallanes } & \\
\hline \multirow{3}{*}{ Michay } & \multirow{3}{*}{ Berberis trigona } & $\mathbf{F}$ & $E$ & $\mathrm{~F}$ & & & & & & & $\mathrm{~N}$ & D & \multirow{3}{*}{$\begin{array}{l}\text { Monoico } \\
\text { Siempreverde }\end{array}$} \\
\hline & & $\mathrm{C}$ & $E$ & $\mathrm{~F}$ & & & & & & & & & \\
\hline & & D & \multicolumn{10}{|c|}{ Maule - Aysén } & \\
\hline \multirow{3}{*}{ Canelo } & \multirow{3}{*}{$\begin{array}{l}\text { Drimys } \\
\text { winteri }\end{array}$} & $\mathbf{F}$ & $\mathrm{E}$ & & & & & A & $S$ & 0 & $\mathrm{~N}$ & D & Monoico, hermafrodita \\
\hline & & C & $\mathrm{E}$ & $\mathrm{F}$ & M & A & & & & & & & Siempreverde \\
\hline & & $\bar{D}$ & & & & & & & & & & & \\
\hline & & $\mathbf{F}$ & & & & & & & $\mathrm{s}$ & 0 & $\mathrm{~N}$ & D & Dioico \\
\hline Maqui & Aristotelia & c & $E$ & & & & & & & & & D & Siempreverde o caducifolio parcial \\
\hline & & D & & & & & & & & & & & \\
\hline & & $\mathrm{F}$ & $\mathrm{E}$ & & & & & & $\mathrm{s}$ & 0 & $\mathrm{~N}$ & D & Monoico, hermafrodita \\
\hline Notro, Ciruelillo & $\begin{array}{l}\text { Embotrium } \\
\text { coccineum }\end{array}$ & C & & $\mathrm{F}$ & M & A & & & & & & & Siempreverde o caducifolio parcial \\
\hline & & D & & & & & & & & & & & \\
\hline & & $\mathbf{F}$ & & & & & & A & $\mathrm{s}$ & 0 & $\mathrm{~N}$ & D & Monoico, poligamo* \\
\hline Maiten & Maytenus boaria & C & $E$ & $\mathrm{~F}$ & $M$ & & & & & & & & Siempreverde \\
\hline & & D & & & & & & & & & & & \\
\hline & & $\mathbf{F}$ & $\mathrm{E}$ & $\mathrm{F}$ & $M$ & $A$ & & & & 0 & $\mathrm{~N}$ & D & Monoico, hermafrodita \\
\hline Avellano chileno & Gevuina & c & $\mathrm{E}$ & $\mathrm{F}$ & M & $A$ & & & & 0 & $\mathrm{~N}$ & D & Siempreverde \\
\hline & & D & & & & & & & & & & & \\
\hline & & $F$ & & & & & & & & & $\mathrm{~N}$ & D & Monoico \\
\hline $\begin{array}{l}\text { Coihue de } \\
\text { Magallanes }\end{array}$ & $\begin{array}{l}\text { Nothofagus } \\
\text { betuloides }\end{array}$ & c & $E$ & $\mathrm{~F}$ & & & & & & & & & Siempreverde \\
\hline & & D & & & & & & & & & & & Producción de mielatos ${ }^{\star *}$ \\
\hline & & $F$ & & & & & & & & & $\mathrm{~N}$ & D & Monoico \\
\hline Lenga & Nothofagus & c & $E$ & $\mathrm{~F}$ & & & & & & & & & Caducifolio \\
\hline & & D & & & & & & & & & & & \\
\hline & & $\mathbf{F}$ & $\mathrm{E}$ & $\mathrm{F}$ & M & A & M & & & & & D & Monoico \\
\hline Arrayan & Luma & $\mathrm{C}$ & & & $M$ & $A$ & $M$ & & & & & & Siempreverde \\
\hline & & D & & & & & & & & & & & \\
\hline & & $F$ & & & & & & & & 0 & $\mathrm{~N}$ & D & Monoico \\
\hline Palo duro, maitén & $\begin{array}{l}\text { Maytenus } \\
\text { magellanica }\end{array}$ & c & $E$ & $\mathrm{~F}$ & & & & & & & & & Siempreverde \\
\hline & & D & & & & & & & & & & & \\
\hline & & $F$ & & & & & & & $\mathrm{~s}$ & 0 & & & Dioico \\
\hline Chaura & $\begin{array}{l}\text { Gaultheria } \\
\text { mucronata }\end{array}$ & c & & & & A & $M$ & & & & & & Siempreverde \\
\hline & & D & & & & & & & & & & & \\
\hline & & $F$ & $\mathrm{E}$ & $\mathrm{F}$ & & & & & & & & D & Monoico, hermafrodita \\
\hline Murta, Murtilla & $\begin{array}{c}\text { Ugni } \\
\text { molinae }\end{array}$ & C & & $\mathrm{F}$ & $M$ & A & & & & & & & Siempreverde \\
\hline & & D & & & & & & & & & & & \\
\hline
\end{tabular}

C: Época cosecha semillas

D: Distribución geográfica (regiones)

* Árbol polígamo-monoico. Puede presentar en un mismo individuo flores masculinas, femeninas y hermafroditas.

${ }^{\star *}$ Miel de mielada, o ligamaza o mielato: Miel producida por abejas pero que procede de exudaciones de las plantas, especialmente plantas caducas y de coníferas. Su color es entre pardo claro y casi negro. Tiene olor intenso y resinoso particular. El contenido de azúcar es igual o superior al $60 \%$. (Diaz, 2003). 
En este calendario del Cuadro $\mathrm{N}^{\circ} 7$ se incluyen especies nativas chilenas, pero también se pueden considerar algunas especies exóticas, que podrían resistir las condiciones ambientales de la región y que pueden producir néctares y mielatos (exudaciones) apropiados para la producción de miel. Entre estas se encuentran eucaliptos resistentes al frío, como Eucalyptus gunni, E. viminalis E. nitens, E. pauciflora, E. dalrymplena y otros; Robinia pseudoacacia, especie de uso tradicional en la apicultura de Hungría, y otras.

\section{CONCLUSIONES}

El enfoque participativo de la propuesta ha sido fundamental para la aceptación y compromiso en las actividades desarrolladas en el proyecto por parte de los apicultores.

Dada la afinidad con las labores agrícolas, se ha apreciado entre los propietarios un alto interés en actividades como la viverización de plantas, la injertación y las técnicas de plantación de especies forestales de utilidad melífera.

El énfasis en el uso de especies forestales nativas para la conformación de huertos melíferos provoca un mayor compromiso y satisfacción en los apicultores porque se sienten aportando en forma real a la conservación sostenida del bosque nativo chileno.

El establecimiento de unidades productivas melíferas, como innovación tecnológica, permitirá a los apicultores disponer de materia prima propia (polen, néctar, agua) para la producción de mieles y otros productos apícolas in situ, no dependiendo de terceros para el establecimiento, mantención y alimentación de sus apiarios. Con esto se espera que sea posible incrementar la cantidad y calidad de sus mieles y eventualmente predecir el tipo de miel a producir en cada temporada, en cuanto a su composición florística y polínica para su comercialización (monoflorales, biflorales, poliflorales) y controlar la calidad de las mieles, adaptándose a los estándares internacionales.

Otras acciones, como la mantención e información oportuna del estado de cada huerto, así como el monitoreo floral de los mismos, son compromisos importantes al que deben concurrir los apicultores para obtener el mayor provecho de esta unidades productivas.

Se detecta que no es suficiente aún la capacitación en el sentido de conseguir el interés de algunos apicultores para convertirse en monitores y capacitadores de otros colegas.

El análisis y caracterización de las mieles de los apicultores de Bio Bio concitó un gran interés por parte de estos, sin embargo deja en evidencia que falta investigación para profundizar ventajas y desventajas de los resultados obtenidos.

En el caso de la Patagonia chilena, de acuerdo al interés que exista por la actividad apícola podrían desarrollarse programas de implementación de unidades melíferas adecuadas a las condiciones climáticas de la región. Estas condiciones climáticas (velocidad y frecuencia del viento, bajas temperaturas, entre otras) desfavorables, sin embargo, se convierten en ventajas comparativas para la producción de mieles únicas y colmenas con un mejor estado sanitario.

\section{REFERENCIAS}

Díaz, C., 2003. Determinación del origen floral y caracterización física y química de mieles de abeja (Apis mellifera L.), etiquetadas como "miel de ulmo" (Eucriphya cordifolia Cav.). Tesis de grado para Licenciado en Agronomía en la Universidad Austral de Chile. 97 pág.

FIA, 2016. Agenda de Innovación Agraria. Apicultura.

Instituto Nacional de Estadística y Censos, 1990. Censo Nacional Agropecuario 1988. República Argentina, Presidencia de la Nación, Secretaría de Planificación. 
En:

https://books.google.cl/books/about/Censo_nacional_agropecuario_1988.html?id=7VloAQAAIAAJ\&redir_esc=y

INE, 2016. Censo Agropecuario 2007. Instituto Nacional de Estadísticas, Chile.

En: http://www.ine.cl/canales/chile_estadistico/censos_agropecuarios/censo_agropecuario_07.php

INTA, 2016. Datos de Producción Apícola. Situación Actual. INDEC, Censos, Encuestas Nacionales, RENAPA, Consejo Asesor Apícola y CEPyME).

En: http://sipan.inta.gov.ar/productos/ssd/vc/neuquen/ap/apicultura_datos.htm

Massaccesi, C., 2002. Apicultura en la Patagonia Andina. Manual INTA. Lago Puelo, abril, 2002.64 p.

Manriqez, A., 2015. Finlandia- La Apicultura Urbana Realizada por Gente Joven. ApiNews.

En: http://www.apinews.com/es/noticias/item/28713-finlandia-la-apicultura-urbana-realizada-por-gente-joven

Manríquez, A, 2015a. Islandia- La Apicultura en el País. ApiNews,

En: http://www.apinews.com/es/noticias/item/28871-islandia-la-apicultura-en-el-pais

Scatturice, L.; Christiansen, R. y Cañari, L., 2005. Apicultura en la Patagonia Argentina. Revista Electrónica de Veterinaria REDVET Vol. VI, № 4, Abril 2005.

En: http://www.veterinaria.org/revistas/redvet/n040405/040510.pdf 